\title{
Commentary: Socioeconomic differences in one-year survival after ischemic stroke: the effect of acute and post-acute care-pathways in a cohort study
}

\author{
Valeria Belleudi, Nera Agabiti*, Marina Davoli, Danilo Fusco \\ 1Department of Epidemiology, Lazio Regional Health Service, Via Cristoforo Colombo, 112, 00147 Rome, Italy
}

Article Info

\section{Article Notes}

Received: June 11, 2018

Accepted: June 28, 2018

*Correspondence:

Dr. Nera Agabiti

Department of Epidemiology, Lazio Regional Health Service

Via Cristoforo Colombo, 112,

00147 Rome, Italy;

Email: n.agabiti@deplazio.it

(c) 2018 Agabiti N. This article is distributed under the terms of the Creative Commons Attribution 4.0 International License
The inverse relationship between risk of dying from a stroke and Socio-Economic Status (SES) has been extensively investigated ${ }^{1-3}$. The mechanisms by which SES affects stroke mortality are not well understood. Worse lifestyles might be associated with higher incidence of stroke, while clinical conditions, typically observed in groups of people with a relatively low socioeconomic position, have been shown to play an important mediating role in the social inequality, even when a patient has access to the health services. However, some studies have shown that adjusting for several risk factors did not explain the entire association between stroke and $\mathrm{SES}^{4-5}$. An alternative reason for this differential could be the heterogeneity in the care-pathway offering. The aim of our study was to explore the role of the care-pathway, ${ }^{6}$ both for the acute and post-acute phases, on the association between education level and one-year survival after admission for ischemic stroke. A cohort of patients with ischemic stroke was extracted from regional health information systems during 2011/2012 (Lazio, Italy about 6 million of inhabitants) and the association between education level and mortality after stroke was studied for acute and post-acute phases. We focused on patients accessing health-care to investigate specifically the effect of different scenarios of quality of care-pathways, identified on the basis of hospital performance, access to rehabilitation and drug treatment post-discharge. The one-year survival probability according to education level and care-pathway scenarios was estimated multiplying the probabilities to survive both acute and post-acute phases. Finally, the one-year survival probability ratios (PRs), university versus elementary education, and their Bootstrap confidence intervals (BCI95\%) were calculated for the best and worst scenarios.

In a cohort of 9,958 patients hospitalized for ischemic stroke the overall 1-year mortality was $26.3 \%$. We found lower mortality among higher educated patients after hospital admission for ischemic stroke both in the acute $(\mathrm{OR}=0.90$; p-trend $<0.001)$ and post-acute phases $(\mathrm{OR}=0.85$; p-trend $<0.001)$. Patients who experienced the best carepathway, in terms of access to a high-performance hospital and to rehabilitation and drug treatment, had a higher 1-year survival. Among the patients who followed the best care-pathway, the socioeconomic associated differential in mortality was lower $(1.06$; 95\%BCI=1.031.10) than among those patients who received the worst care-pathway $(1.17 ; 95 \% \mathrm{BCI}=1.09-1.25)$. Our study suggests that this differential is dependent, at least in part, on the care-pathway. Other studies have proposed this theory, but it remains controversial.

The key messages of our study can be summarized as follows. 
Describing the real-life adherence to evidence-based pathways of care and measuring the impact of health care practices using observational study designs have a great potential to inform both scientific community and administrators and guide strategies and interventions to promote improvement in the quality of care. In the case of a relevant disease such as stroke, the whole emergency care system is called to invest all efforts to guarantee timely and appropriate care to the patients. Access to evidencebased treatment in stroke units as well as early initiation of rehabilitation interventions are associated with a better long-term prognosis in most cases ${ }^{7}$. Moreover, adherence to pharmacological treatment after stroke is a key component of the stroke patients' management. The NICE guidelines recommend the use of both anticoagulant and antiplatelet drugs. The use of antihypertensive and statins has been shown to have beneficial effects ${ }^{8,9}$. The optimal pharmacological strategy remains controversial. Mechanisms involved in differential one-year survival after stroke in populations with different socioeconomic levels are complex, and depend on geographical and cultural contest. In Lazio Region, based on a regional law a hub\&spoke integrated care for stroke patients is present since 2015, with four "high volume" hospitals as hub to treat more serious patients. The Regional Outcomes Evaluation Program (P.Re.Val.E.) annually calculate and publicly diffuse data on quality of care using indicators for many medical and surgical conditions, including stroke, on the basis of health information system linked databases, to monitor the effectiveness of the integrated pathway of care in clinical practice and the impact of implementation of new organizational health- care models. The evidence of reduced survival in stroke patients with low socioeconomic status does confirm the role of clinical behavioural and contextual risk factors but also underlines the importance of consider potential inequity in the provision of care, that is not expected in a universal coverage health system like in Italy. In our Region, we are particularly interested in systematically monitoring quality indicators, on behalf of P.Re.Val.E., under the so-called equity-lent approach, ${ }^{10}$ that is calculating indicators, for acute and chronic conditions, stratified by different SES groups ${ }^{11}$. Moreover, based on results from our study, we wish that Audit and Feedback interventions - quality improvement strategies considered effective in improving quality of care ${ }^{12-13}$ would be more frequent in health care organizations. In general, there is a need to promote the culture of measurement as the first step to improve performance, among clinicians and health-care operators, and to specifically analyse and discuss potential inequity in the care provision. The fact that the gap in survival after stroke between low and high socioeconomic status is less evident in the populations exposed to the optimal care pathways is a very important point. In fact, the promotion of high quality of care in the overall population may lead to an improvement in health for disadvantaged people. This represents a great challenge for both administrators, health professionals, and researchers.

\section{References}

1. Arrich J, Lalouschek W, Müllner M. Influence of socioeconomic status on mortality after stroke: retrospective cohort study. Stroke. 2005 36: 310-4.

2. Cesaroni G, Agabiti N, Forastiere F, et al. Socioeconomic differences in stroke incidence and prognosis under a universal healthcare system. Stroke. 2009; 40: 2812-9.

3. Ahacic K, Trygged S, Kåreholt I. Income and education as predictors of stroke mortality after the survival of a first stroke. Stroke Res Treat. 2012; 2012: 983145

4. Kuper H, Adami HO, Theorell T, et al. The socioeconomic gradient in the incidence of stroke: a prospective study in middle-aged women in Sweden. Stroke. 2007; 38: 27-33.

5. Brown P, Guy M, Broad J. Individual socio-economic status, community socio-economic status and stroke in New Zealand: a case control study. Soc Sci Med. 2005; 61: 1174-88

6. Belleudi V, Sciattella P, Agabiti N, et al. Socioeconomic differences in one-year survival after ischemic stroke: the effect of acute and postacute care-pathways in a cohort study. BMC Public Health. 2016 May 16; 16: 408. doi: 10.1186/s12889-016-3019-8.

7. Royal College of Physicians, National Collaborating Centre for Chronic Conditions (UK). Stroke: National Clinical Guideline for Diagnosis and Initial Management of Acute Stroke and Transient Ischaemic Attack (TIA). London: Royal College of Physicians of London; 2008.

8. Squizzato A, Romualdi E, Dentali F, et al. Statins for acute ischemic stroke. Cochrane Database Syst Rev. 2011; 10: CD007551.

9. Andrew NE, Kim J, Thrift AG, et al. Prescription of antihypertensive medication at discharge influences survival following stroke. Neurology. 2018 Feb 27; 90(9): e745-e753.

10. Bargagli AM, Agabiti N, Davoli M. Applying an equity lens approach to evaluate the effects of health interventions. Epidemiol Prev. 2014 Mar-Apr; 38(2): 82-3.

11. Ventura M, Fusco D, Bontempi K, et al. Regional Outcome Evaluation Program (P.Re.Val.E.): Reduction of inequality in access to effective health care in the Lazio region of Italy (2012-2015). PLoS One. 2018 Mar 27; 13(3): e0194972. doi: 10.1371/journal.pone.0194972. eCollection 2018

12. Ivers NM1, Grimshaw JM, Jamtvedt G, et al. Growing literature, stagnant science? Systematic review, meta-regression and cumulative analysis of audit and feedback interventions in health care. J Gen Intern Med. 2014 Nov; 29(11): 1534-41. doi: 10.1007/s11606-0142913-y.

13. Brehaut JC, Colquhoun HL, Eva KW, et al. Practice Feedback Interventions: 15 Suggestions for Optimizing Effectiveness. Ann Intern Med. 2016 Mar 15; 164(6): 435-41. doi: 10.7326/M15-2248. Epub 2016 Feb 23. 